\title{
THE LOCAL DENSITY AND THE LOCAL WEAK DENSITY IN THE SPACE OF PERMUTATION DEGREE AND IN HATTORI SPACE
}

\author{
Tursun K. Yuldashev ${ }^{\dagger}$, Farkhod G. Mukhamadiev ${ }^{\dagger \dagger}$ \\ National University of Uzbekistan, \\ 700174, Tashkent, Uzbekistan \\ †tursun.k.yuldashev@gmail.com, ††farhod8717@mail.ru
}

\begin{abstract}
In this paper, the local density $(l d)$ and the local weak density $(l w d)$ in the space of permutation degree as well as the cardinal and topological properties of Hattori spaces are studied. In other words, we study the properties of the functor of permutation degree $S P^{n}$ and the subfunctor of permutation degree $S P_{G}^{n}$, $P$ is the cardinal number of topological spaces. Let $X$ be an infinite $T_{1}$-space. We prove that the following propositions hold.

(1) Let $Y^{n} \subset X^{n}$; (A) if $d\left(Y^{n}\right)=d\left(X^{n}\right)$, then $d\left(S P^{n} Y\right)=d\left(S P^{n} X\right)$; (B) if $l w d\left(Y^{n}\right)=l w d\left(X^{n}\right)$, then $l w d\left(S P^{n} Y\right)=l w d\left(S P^{n} X\right)$.

(2) Let $Y \subset X$; (A) if $l d(Y)=l d(X)$, then $l d\left(S P^{n} Y\right)=l d\left(S P^{n} X\right)$; (B) if $w d(Y)=w d(X)$, then $w d\left(S P^{n} Y\right)$ $=w d\left(S P^{n} X\right)$.

(3) Let $n$ be a positive integer, and let $G$ be a subgroup of the permutation group $S_{n}$. If $X$ is a locally compact $T_{1}$-space, then $S P^{n} X, S P_{G}^{n} X$, and $\exp _{n} X$ are $k$-spaces.

(4) Let $n$ be a positive integer, and let $G$ be a subgroup of the permutation group $S_{n}$. If $X$ is an infinite $T_{1}$-space, then $n \pi w(X)=n \pi w\left(S P^{n} X\right)=n \pi w\left(S P_{G}^{n} X\right)=n \pi w\left(\exp _{n} X\right)$.

We also have studied that the functors $S P^{n}, S P_{G}^{n}$, and $\exp _{n}$ preserve any $k$-space. The functors $S P^{2}$ and $S P_{G}^{3}$ do not preserve Hattori spaces on the real line. Besides, it is proved that the density of an infinite $T_{1}$-space $X$ coincides with the densities of the spaces $X^{n}, S P^{n} X$, and $\exp _{n} X$. It is also shown that the weak density of an infinite $T_{1}$-space $X$ coincides with the weak densities of the spaces $X^{n}, S P^{n} X$, and $\exp _{n} X$.
\end{abstract}

Keywords: Local density, Local weak density, Space of permutation degree, Hattori space, Covariant functors.

\section{Introduction}

In mathematical research in the modern world, a special place is occupied by the study of the topological properties of objects in various topological spaces. Research in general topology is topical, where the properties of topological spaces and their continuous mappings, operations on topological spaces and their mappings, as well as the classification of topological spaces are studied. This section of general topology uses concepts such as neighborhood, closure, compactness, density, separability, cardinal number, $\pi$-base of sets, sum, intersection, Tikhonov product, and others. An overview of the main stages in the development of set-theoretic topology is given in [1]. Some cardinal properties of topological spaces related to weak density were studied in [4]. In [5], some cardinal properties of Hattori spaces and their hyperspaces were studied. In [2,6], some properties of topological spaces related to local density and local weak density in various topological spaces were studied.

Along with the concepts of local $\tau$-density and local weak $\tau$-density in various topological spaces, we are interested in such concepts as hereditary Souslin number, hereditary density, hereditary $\pi$ weight, hereditary Shanin number, hereditary pre-Shanin number, hereditary caliber, hereditary precaliber, hereditary weak density, hereditary Lindelöf number, and hereditary extent of topological spaces. 
Denote by $P$ the cardinal number of topological spaces. Let $S P^{n}$ be a functor of permutation degree, and let $S P_{G}^{n}$ be a subfunctor of the functor of permutation degree.

At the Prague topological symposium in 1981, V.V. Fedorchuk posed the following general problem in the theory of covariant functors [8] and thus created a new direction of research in this area of topology.

Problems. Let $P$ be a geometrical or topological property, and let $F$ be a covariant functor. If $X$ has the property $P$, does $F(X)$ have the same property $P$ ? The opposite problem: for which functors $F(X)$ the space $X$ has the property $P$ if $F(X)$ has this property?

In [2], it was proved that the property of local density and the property of local weak density coincide for stratifiable spaces. These cardinal numbers are preserved under open mappings and are inverse invariant of a class of closed irreducible mappings.

In our present work, we prove that the following propositions are true for an infinite $T_{1}$-space $X$ :

(1) if $l d(Y)=l d(X)$ for $Y \subset X$, then $l d\left(S P^{n} Y\right)=l d\left(S P^{n} X\right)$;

(2) if $l w d(Y)=l w d(X)$ for $Y \subset X$, then $l w d\left(S P^{n} Y\right)=l w d\left(S P^{n} X\right)$;

(3) if $X$ is a locally compact $T_{1}$-space, $n$ is a positive integer, and $G$ is a subgroup of the permutation group $S_{n}$, then $S P^{n} X, S P_{G}^{n} X$, and $\exp _{n} X$ are $k$-spaces;

(4) if $X$ is an infinite $T_{1}$-space, $n$ is a positive integer, and $G$ is a subgroup of the permutation group $S_{n}$, then

$$
n \pi w(X)=n \pi w\left(S P^{n} X\right)=n \pi w\left(S P_{G}^{n} X\right)=n \pi w\left(\exp _{n} X\right) .
$$

We also prove that the functors $S P^{2}$ and $S P_{G}^{3}$ do not preserve Hattori spaces on the real line. In addition, we prove that the density of an infinite $T_{1}$-space $X$ coincides with the densities of the spaces $X^{n}, S P^{n} X$, and $\exp _{n} X$. We show that the weak density of an infinite $T_{1}$-space $X$ coincides with the weak densities of the spaces $X^{n}, S P^{n} X$, and $\exp _{n} X$.

\section{Auxiliary material}

Recall some notation, definitions, and statements that are widely used in this paper. The permutation group of $X$ is the group of all permutations (one-to-one and onto mappings $X \rightarrow X$ ). The permutation group of a set $X$ is usually denoted by $S(X)$. If $X=\{1,2,3, \ldots, n\}$, then $S(X)$ is denoted by $S_{n}$.

Let $X^{n}$ be the $n$th power of a compact set $X$. The permutation group $S_{n}$ of all permutations acts on the $n$th power $X^{n}$ as the permutation of coordinates. The set of all orbits of this action with quotient topology is denoted by $S P^{n} X$. Thus, points of the space $S P^{n} X$ are finite subsets (equivalence classes) of the product $X^{n}$. Thus, two points $\left(x_{1}, x_{2}, \ldots, x_{n}\right),\left(y_{1}, y_{2}, \ldots, y_{n}\right) \in X^{n}$ are equivalent if there is a permutation $\sigma \in S_{n}$ such that $y_{i}=x_{\sigma(i)}$. The space $S P^{n} X$ is called the $n$-permutation degree of a space $X$. An equivalent relation by which we obtain the space $S P^{n} X$ is called the symmetric equivalence relation. The $n$th permutation degree is always a quotient of $X^{n}$. Thus, the quotient mapping is denoted as $\pi_{n}^{s}: X^{n} \rightarrow S P^{n} X$, where $\pi_{n}^{s}\left(\left(x_{1}, x_{2}, \ldots, x_{n}\right)\right)=[x=$ $\left.\left(x_{1}, x_{2}, \ldots, x_{n}\right)\right]$ is an orbit of the point $x=\left(x_{1}, x_{2}, \ldots, x_{n}\right) \in X^{n}$.

The concept of permutation degree has generalizations. Let $S P_{G}^{n} X$ be any subgroup of the group $S_{n}$. Then it also acts on $X^{n}$ as the group of permutations of coordinates. Consequently, it generates a $G$-symmetric equivalence relation on $X^{n}$. This quotient space of the product $X^{n}$ under the $G$-symmetric equivalence relation is called the $G$-permutation degree of the space $X$ and is denoted by $S P_{G}^{n} X$. The operation $S P_{G}^{n}$ is also a covariant functor in the category of compact sets and is said to be a functor of $G$-permutation degree. If $G=S_{n}$, then $S P_{G}^{n}=S P^{n}$. If the group $S P_{G}^{n} X$ consists of only one element, then $S P_{G}^{n} X=X^{n}$. 
Let $X$ be a $T_{1}$-space. The collection of all nonempty closed subsets of $X$ is denoted by $\exp X$. The family $B$ of all sets of the form

$$
O\left\langle U_{1}, \ldots, U_{n}\right\rangle=\left\{F: F \in \exp X, F \subset \bigcup_{i=1}^{n} U_{i}, F \cap U_{i} \neq \emptyset, i=1,2, \ldots, n\right\}
$$

generates a topology on the set $\exp X$, where $U_{1}, \ldots, U_{n}$ is a family of open sets of $X$. This topology is called the Vietoris topology. The set $\exp X$ with the Vietoris topology is called the exponential space or the hyperspace of $X$ [9]. Let $X$ be a $T_{1}$-space. Denote by $\exp _{n} X$ the set of all closed subsets of $X$ such that $\exp _{n} X=\{F \in \exp X:|F| \leq n\}$.

We use the following notation:

$$
\exp _{\omega} X=\cup\left\{\exp _{n} X: n=1,2, \ldots\right\}, \quad \exp _{c} X=\{F \in \exp X: F \text { is compact in } X\} .
$$

It is clear that $\exp _{n} X \subset \exp _{\omega} X \subset \exp _{c} X \subset \exp X$ for any topological space $X$. Moreover, if $G_{1} \subset G_{2}$ for subgroups $G_{1}$ and $G_{2}$ of the permutation group

$$
\pi_{n}^{s}\left(\left(x_{1}, x_{2}, \ldots, x_{n}\right)\right)=\left[x=\left(x_{1}, x_{2}, \ldots, x_{n}\right)\right] \in X^{n},
$$

then we have the following chain of factorizations of functors [9]:

$$
X^{n} \rightarrow S P_{G_{1}}^{n} X \rightarrow S P_{G_{2}}^{n} X \rightarrow S P^{n} X \rightarrow \exp _{n} X .
$$

A subset $D$ of a topological space $X$ is called a dense set in $X$ if $[D]=X$. Define the density $d(X)$ of $X$ by $d(X)=\min \{|D|: D$ is a dense subset of $X\}$ [7].

We say that the local density of a topological space $X$ is $\tau$ at a point $x$ if $\tau$ is the smallest cardinal number such that $x$ has a neighbourhood of density $\tau$ in $X$. The local density at a point $x$ is denoted by $l d(x)$. The local density of a topological space $X$ is defined as the supremum of all numbers $l d(x)$ for $x \in X: l d(X)=\sup \{l d(x): x \in X\}[2,6]$. It is known that $l d(X) \leq d(X)$ for any topological space.

Example. Let $\mathbb{R}$ be the real line with discrete topology. In the discrete topological space $\left(\mathbb{R}, \tau_{d}\right)$, every point $x \in \mathbb{R}$ has the one-point neighbourhood $\{x\}$. It follows that $l d\left(\mathbb{R}, \tau_{d}\right)=1$. On the other hand, the boundary set of any set is empty in a discrete space, and hence the only dense set is the space itself. This means that $d\left(\mathbb{R}, \tau_{d}\right)=|\mathbb{R}|=c$. Then $1=l d\left(\mathbb{R}, \tau_{d}\right)<d\left(\mathbb{R}, \tau_{d}\right)=c$.

We say that the weak density of a topological space is $\tau \geq \aleph_{0}$ if $\tau$ is the smallest cardinal number such that there exists a $\pi$-base coinciding with $\tau$ centered systems of open sets, i.e., there is a $\pi$-base $B=\bigcup\left\{B_{\alpha}: \alpha \in A\right\}$, where $B_{\alpha}$ is a centered system of open sets for every $\alpha \in A$, $|A|=\tau$.

The weak density of a topological space $X$ is denoted by $w d(X)$. If $d(X)=\tau \geq \aleph_{0}$, then $w d(X) \leq \tau$. Similarly, if $Y$ is dense in a topological space $X$, then $w d(Y)=w d(X)$ [3]. The following theorem and proposition were proved in [3].

Theorem 1. Let $\left\{X_{\alpha}: \alpha \in A\right\}$ be a family of topological spaces such that $w d\left(X_{\alpha}\right) \leq \tau \geq \aleph_{0}$ for every $\alpha \in A$, where $|A| \leq 2^{\tau}$. Then $w d\left(\prod_{\alpha \in A} X_{\alpha}\right) \leq \tau$.

Proposition 1. Assume that $X$ and $Y$ are topological spaces and there exists a continuous "onto" mapping $f: X \rightarrow Y$. Then $w d(Y) \leq w d(X)$.

A topological space $X$ is called a locally weak $\tau$-dense space at a point $x \in X$ if $\tau$ is the smallest cardinal number such that $x$ has a neighbourhood of weak density $\tau$ in $X$. The local weak density at a point $x$ is denoted by $l w d(x)$. The local weak density of a topological space $X$ is defined as the supremum of all numbers $l w d(x)$ for $x \in X: \operatorname{lwd}(X)=\sup \{\operatorname{lwd}(x): x \in X\}$ [2,6]. If $X$ is a space of local density $\tau$ and $f: X \rightarrow Y$ is an open continuous "onto" mapping, then $Y$ is a space of local density $\tau$ [12]. The quotient mapping $\pi_{n}^{s}: X^{n} \rightarrow S P^{n} X$ is a clopen continuous onto mapping [13].

The following two statements are from [11]. 
Proposition 2. If $X$ is a topological space, then $\exp _{n} X$ is dense in $\exp X$.

Proposition 3. $X$ is separable if and only if $\exp X$ is separable.

These propositions imply that, for any infinite $T_{1}$-space $X$, we have

$$
\operatorname{lwd}(X)=\operatorname{lwd}\left(X^{n}\right)=\operatorname{lwd}\left(S P^{n} X\right) .
$$

The following theorem was proved in [4].

Theorem 2. Let $X$ be an infinite $T_{1}$-space. Then $w d(X)=w d\left(\exp _{n} X\right)=w d(\exp X)$.

To substantiate our results, we also use the following notation and definitions from [7].

An uncountable cardinal number $\tau$ is a caliber of a topological space if every family of cardinality $\tau$ consisting of nonempty open sets contains subfamily of the same cardinality with nonempty intersection. The caliber of a topological space $X$ is denoted by $k(X)$.

The cardinal number $\min \left\{\tau: \tau^{+}\right.$is a caliber of $\left.X\right\}$ is called the Shanin number of $X$ and is denoted by $\operatorname{sh}(X)$.

A cardinal number $\tau>\aleph_{0}$ is called a precaliber of a space $X$ if every family of cardinality $\tau$ consisting of nonempty open subsets of $X$ contains a subfamily of cardinality $\tau$ with finite intersection. Define

$$
p k(X)=\left\{\tau^{+}: \tau \text { is a precaliber of } X\right\} .
$$

The cardinal number $\operatorname{psh}(X)=\min \left\{\tau^{+}: \tau\right.$ is a precaliber of $\left.X\right\}$ is called the pre-Shanin number. We always have $c(X) \leq p \operatorname{sh}(X) \leq \operatorname{sh}(X) \leq d(X)$.

The Lindelöf number $l(X)$ of $X$ is defined as $l(X)=\min \{\tau$ : every open cover of $X$ has a refinement of cardinality $\leq \tau\}+\aleph_{0}$. If $l(X)=\aleph_{0}$, i.e., every open cover has a countable refinement, we say that $X$ is a Lindelöf space.

The notion of cellularity (Souslin number) $c(X)$ of $X$ is defined as $c(X)=\min \{\tau$ : every family of pairwise disjoint nonempty open subsets of $X$ has cardinality $\leq \tau\}+\aleph_{0}$. If $c(X)=\aleph_{0}$, we say that $X$ has the countable chain condition (Souslin property).

The spread $s(X)$ and the extent $e(X)$ are defined as follows: $s(X)=\sup \{|D|: D$ is a discrete subset of $X\}+\aleph_{0}$ and $e(X)=\sup \{|D|: D$ is a discrete closed subset of $X\}+\aleph_{0}$, respectively.

For a metrizable space $X$, we have $l(X)=d(X)=c(X)=s(X)=e(X)$.

For a cardinal function $\varphi$, we define the corresponding hereditary cardinal function $h \varphi=$ $\sup \{\varphi(Y): Y \subset X\}$. For example, we have the hereditary Souslin number $h c(X)$, the hereditary density $h d(X)$, the hereditary $\pi$-weight $h \pi w(X)$, and the hereditary Shanin number $h s h(X)$. Similar symbols we use to denote the hereditary pre-Shanin number, the hereditary caliber, the hereditary precaliber, the hereditary weak density, the hereditary Lindelöf number, and the hereditary extent of the space $X$, respectively: $h p s h(X), h k(X), h p k(X), h w d(X), h l(X)$, and $h e(X)$.

It is easy to see that the hereditary Souslin number $h c(X)$ of a space $X$ coincides with its spread $s(X)$.

Definition 1. A topological space is a $k$-space if it is a quotient image of some topological space $Y$.

Recall that a topological space is locally compact if, for every $x \in X$, there exists a neighbourhood $U$ of $x$ such that $[U]$ is a compact subspace of $X$.

In 2010, Hattori defined [10] the following topology on $\mathbb{R}$. Let $\mathbb{R}$ be the real line and $\mathbb{A} \subseteq \mathbb{R}$. The topology $\tau(\mathbb{A})$ on $\mathbb{R}$ is defined as follows: 
(1) for each $x \in \mathbb{A},\{(x-\varepsilon, x+\varepsilon): \varepsilon>0\}$ is the neighbourhood base at $x$;

(2) for each $x \in \mathbb{R} \backslash \mathbb{A},\{[x, x+\varepsilon): \varepsilon>0\}$ is the neighbourhood base at $x$.

The space $(\mathbb{R}, \tau(\mathbb{A}))$ is called [5] a Hattori space. Let $\tau_{\mathbb{E}}$ be the Euclidean topology on $\mathbb{R}$. Note that, for any $\mathbb{A}, \mathbb{B} \subseteq \mathbb{R}$, we have $\mathbb{A} \supseteq \mathbb{B}$ if $\tau(\mathbb{A}) \subseteq \tau(\mathbb{B})$, in particular, $\tau(\mathbb{R})=\tau_{\mathbb{E}} \subseteq \tau(\mathbb{A})$ and $\tau(\mathbb{B}) \subseteq \tau(\emptyset)=\tau_{S}$. We set $P_{\text {top }}(\mathbb{R})=\{\tau(\mathbb{A}): \mathbb{A} \subseteq \mathbb{R}\}$ and define a partial order $\leq$ on $P_{\text {top }}(\mathbb{R})$ by the inclusion: $\tau(\mathbb{A}) \leq \tau(\mathbb{B})$ if $\tau(\mathbb{A}) \subseteq \tau(\mathbb{B})$.

\section{Main results}

Theorem 3. Let $X$ be an infinite $T_{1}$-space, and let $Y^{n}$ be dense in $X^{n}$. Then $S P^{n} Y$ is also dense in $S P^{n} X$.

P r o o f. Let $Y^{n}$ be a dense subset of $X^{n}$, and let $S P^{n} U$ be an arbitrary open set from $S P^{n} X$. Since the mapping $\pi_{n}^{s}: X^{n} \rightarrow S P^{n} X$ is continuous, the set $\left(\pi_{n}^{s}\right)^{-1}\left(S P^{n} U\right) \subset X^{n}$ is open. Thus, taking into account the density of $Y^{n}$ in $X^{n}$, we conclude that $\left(\pi_{n}^{s}\right)^{-1}\left(S P^{n} U\right) \cap Y^{n} \neq \emptyset$. Therefore, there exists $y \in Y^{n}$ such that $y \in\left(\pi_{n}^{s}\right)^{-1}\left(S P^{n} U\right)$. Then $\pi_{n}^{s}(y) \in S P^{n} U$ (and $\pi_{n}^{s}(y) \in S P^{n} Y$ ). Hence, we have $S P^{n} U \cap S P^{n} Y \neq \emptyset$ for every open set $S P^{n} U$. This means that the set $S P^{n} Y$ is dense in $S P^{n} X$. Theorem 3 is proved.

Corollary 1. If $X$ is an infinite $T_{1}$-space and $Y^{n}$ is a subset of $X^{n}$ such that $d\left(Y^{n}\right)=d\left(X^{n}\right)$, then $d\left(S P^{n} Y\right)=d\left(S P^{n} X\right)$.

Proposition 4. Assume that $X$ is an infinite $T_{1}$-space, $n$ is a positive number, and $G_{1}$ and $G_{2}$ are subgroups of the permutation group $S_{n}$ such that $G_{1} \subset G_{2}$. Then

$$
d(X)=d\left(X^{n}\right)=d\left(S P_{G_{1}}^{n} X\right)=d\left(S P_{G_{2}}^{n} X\right)=d\left(S P^{n} X\right)=d\left(\exp _{n} X\right) .
$$

P r o o f. Let $X$ be an infinite $T_{1}$-space. Taking into account that

$$
X^{n} \rightarrow S P_{G_{1}}^{n} X \rightarrow S P_{G_{2}}^{n} X \rightarrow S P^{n} X \rightarrow \exp _{n} X
$$

and the fact that continuous mappings do not increase the density of topological spaces, we directly obtain the inequalities

$$
d(X) \geq d\left(X^{n}\right) \geq d\left(S P_{G_{1}}^{n} X\right) \geq d\left(S P_{G_{2}}^{n} X\right) \geq d\left(S P^{n} X\right) \geq d\left(\exp _{n} X\right) .
$$

By Propositions 2 and 3 , we get $d(X)=d\left(\exp _{n} X\right)$, and hence

$$
d(X)=d\left(X^{n}\right)=d\left(S P_{G_{1}}^{n} X\right)=d\left(S P_{G_{2}}^{n} X\right)=d\left(S P^{n} X\right)=d\left(\exp _{n} X\right) .
$$

Proposition 4 is proved.

Theorem 4. Let $X$ be an infinite $T_{1}$-space, and let $Y^{n}$ be a locally dense set in $X^{n}$. Then $S P^{n} Y$ is also locally dense in $S P^{n} X$.

P r o o f. The set $Y^{n}$ is locally dense in $X^{n}$. By definition, for any point $y \in Y^{n}$, there exists a neighbourhood $O y \subset X^{n}$ such that $O y$ is dense in $X^{n}$. Then Theorem 3 implies that $S P^{n}(O y)$ is also dense in $S P^{n} X$. On the other hand, the quotient mapping $\pi_{n}^{s}: X^{n} \rightarrow S P^{n} X$ is an open mapping. Therefore, $S P^{n}(O y)$ is a neighbourhood of the point $\pi_{n}^{s}(y) \in S P^{n} Y$. Then $S P^{n} Y$ is locally dense in $S P^{n} X$. Theorem 4 is proved. 
Corollary 2. If $X$ is an infinite $T_{1}$-space and $Y \subset X$ is such that $l d(Y)=l d(X)$, then

$$
l d\left(S P^{n} Y\right)=l d\left(S P^{n} X\right) .
$$

Theorem 5. Let $X$ be an infinite $T_{1}$-space. Then $w d(X)=w d\left(S P^{n} X\right)$.

P r o o f. First, we will show that $w d\left(S P^{n} X\right) \leq w d(X)$. Suppose that $w d(X)=\tau \geq \aleph_{0}$. Then $w d\left(X^{n}\right)=\tau$ by Theorem 1. The space $S P^{n} X$ is a continuous image of the space $X^{n}$. Proposition 1 implies that $w d\left(S P^{n} X\right) \leq \tau$.

Now we will prove that $w d\left(S P^{n} X\right) \geq w d\left(X^{n}\right)$. To this end, assume that $w d\left(S P^{n} X\right)=\tau \geq$ $\aleph_{0}$. This means that there exists $S P^{n} B=\cup\left\{S P^{n} B_{\alpha}: \alpha \in A,|A|=\tau\right\}$ and this is a $\pi$-base in $S P^{n} X$, where $S P^{n} B_{\alpha}=\left\{S P^{n} U_{s}^{\alpha}: s \in A_{\alpha}\right\}$ is a centered system of nonempty open sets for every $\alpha \in A$.

We set

$$
B_{\alpha}=\left\{\left(\pi_{n}^{s}\right)^{-1}\left(S P^{n} U_{s}^{\alpha}\right): s \in A_{\alpha}\right\}, \quad B=\cup\left\{B_{\alpha}: \alpha \in A\right\} .
$$

Let us show that $B_{\alpha}$ is a centered system of nonempty open sets in $X^{n}$ for every $\alpha \in A$. For every finite subfamily $\left\{S P^{n} U_{s_{i}}^{\alpha}\right\}_{i=1}^{k}$ of $S P^{n} B_{\alpha}$, we have $\cap_{i=1}^{k} S P^{n} U_{s_{i}}^{\alpha} \neq \emptyset$. Then

$$
\emptyset \neq\left(\pi_{n}^{s}\right)^{-1}\left(\cap_{i=1}^{k} S P^{n} U_{s_{i}}^{\alpha}\right)=\cap_{i=1}^{k}\left(\left(\pi_{n}^{s}\right)^{-1}\left(S P^{n} U_{s_{i}}^{\alpha}\right)\right) .
$$

This shows that $B_{\alpha}=\left\{\left(\pi_{n}^{s}\right)^{-1}\left(S P^{n} U_{s}^{\alpha}\right): s \in A_{\alpha}\right\}$ is also a centered system of nonempty open sets in $X^{n}$. Now, we show that $B$ is a $\pi$-base in $X^{n}$. Since

$$
S P^{n} B=\cup\left\{S P^{n} B_{\alpha}: \alpha \in A,|A|=\tau\right\}
$$

is a $\pi$-base of $S P^{n} X$, for every open subset $S P^{n} U$ of $S P^{n} X$, there exists $S P^{n} U_{s}^{\alpha} \in S P^{n} B_{\alpha} \subset S P^{n} B$ such that $S P^{n} U_{s}^{\alpha} \subset S P^{n} U$. Since the quotient mapping $\pi_{n}^{s}: X^{n} \rightarrow S P^{n} X$ is open and onto, we have

$$
\left(\pi_{n}^{s}\right)^{-1}\left(S P^{n} U_{s}^{\alpha}\right) \subset\left(\pi_{n}^{s}\right)^{-1}\left(S P^{n} U\right)
$$

This means that $B$ is a $\pi$-base in $X^{n}$. Therefore, we have $w d\left(X^{n}\right) \leq \tau$. Theorem 5 is proved.

Corollary 3. If $X$ is an infinite $T_{1}$-space and $Y \subset X$ is such that $w d(Y)=w d(X)$, then

$$
w d\left(S P^{n} Y\right)=w d\left(S P^{n} X\right) .
$$

Theorem 6. Let $X$ be an infinite $T_{1}$-space, and let $Y^{n}$ be locally weakly dense in $X^{n}$. Then $S P^{n} Y$ is locally weakly dense in $S P^{n} X$.

P r o o f. Suppose that $X$ is an infinite $T_{1}$-space and $Y^{n} \subset X^{n}$ is locally weakly dense. Then, for every point $y \in Y^{n}$, there exists a neighbourhood $O y$ such that $O y$ is weakly dense in $X^{n}$. According to Theorem 5, $S P^{n}(O y)=\left\{\pi_{n}^{s}\left(y^{\prime}\right): y^{\prime} \in O y\right\}$ is also weakly dense in $S P^{n} X$. This means that, for every point $\pi_{n}^{s}(y) \in S P^{n} Y$, there exists $S P^{n}(O y)$ such that it is weakly dense in $S P^{n} X$. This shows that $S P^{n} Y$ is locally weakly dense in $S P^{n} X$. Theorem 6 is proved.

Corollary 4. If $X$ is an infinite $T_{1}$-space and $Y^{n} \subset X^{n}$ is such that lwd $\left(Y^{n}\right)=$ lwd $\left(X^{n}\right)$, then lwd $\left(S P^{n} Y\right)=$ lwd $\left(S P^{n} X\right)$.

Proposition 5. Assume that $X$ is an infinite $T_{1}$-space, $n$ is a positive number, and $G_{1}$ and $G_{2}$ are subgroups of the permutation group $S_{n}$ such that $G_{1} \subset G_{2}$. Then

$$
w d(X)=w d\left(X^{n}\right)=w d\left(S P_{G_{1}}^{n} X\right)=w d\left(S P_{G_{2}}^{n} X\right)=w d\left(S P^{n} X\right)=w d\left(\exp _{n} X\right) .
$$


P r o o f. Let $X$ be an infinite $T_{1}$-space. Taking into account that

$$
X^{n} \rightarrow S P_{G_{1}}^{n} X \rightarrow S P_{G_{2}}^{n} X \rightarrow S P^{n} X \rightarrow \exp _{n} X
$$

and the fact that continuous mappings do not increase the weak density of topological spaces, we directly obtain the inequalities

$$
w d(X) \geq w d\left(X^{n}\right) \geq w d\left(S P_{G_{1}}^{n} X\right) \geq w d\left(S P_{G_{2}}^{n} X\right) \geq w d\left(S P^{n} X\right) \geq w d\left(\exp _{n} X\right) .
$$

According to Theorem $2, w d(X)=w d\left(\exp _{n} X\right)$. Hence, we get

$$
w d(X)=w d\left(X^{n}\right)=w d\left(S P_{G_{1}}^{n} X\right)=w d\left(S P_{G_{2}}^{n} X\right)=w d\left(S P^{n} X\right)=w d\left(\exp _{n} X\right) .
$$

Proposition 5 is proved.

Proposition 6. Assume that $X$ is a locally compact $T_{1}$-space, $n$ is a positive integer, and $G$ is a subgroup of the permutation group $S_{n}$. Then $S P^{n} X, S P_{G}^{n} X$, and $\exp _{n} X$ are $k$-spaces.

P r o o f. Let $X$ be a locally compact $T_{1}$-space. Then $X^{n}$ is a locally compact space for each $n \in \mathbb{N}$. The spaces $S P^{n} X, S P_{G}^{n} X$, and $\exp _{n} X$ become quotient images of the space $X^{n}$. Therefore, $S P^{n} X, S P_{G}^{n} X$, and $\exp _{n} X$ are $k$-spaces. Proposition 6 is proved.

Corollary 5. The functors $S P^{n}, S P_{G}^{n}$, and $\exp _{n}$ preserve any $k$-space.

Proposition 7. Assume that $X$ is an infinite $T_{1}$-space, $n$ is a positive integer, and $G$ is a subgroup of the permutation group $S_{n}$. Then $n \pi w\left(S P^{n} X\right)=n \pi w(X)$.

P r o o f. It was proved in Proposition 4 that $d\left(S P^{n} X\right)=d(X), n \in \mathbb{N}$. It is known that any dense set $M \subset X$ can be a $\pi$-net of this space. Hence, we have $n \pi w\left(S P^{n} X\right)=n \pi w(X)$. Proposition 7 is proved.

Corollary 6. Assume that $X$ is an infinite $T_{1}$-space, $n$ is a positive integer, and $G$ is a subgroup of the permutation group $S_{n}$. Then

$$
\begin{gathered}
n \pi w(X)=n \pi w\left(S P^{n} X\right)=n \pi w\left(S P_{G}^{n} X\right)=n \pi w\left(S P_{G_{1}}^{n} X\right)= \\
=n \pi w\left(S P_{G_{2}}^{n} X\right)=n \pi w\left(\exp _{n} X\right)=n \pi w\left(\exp _{\omega} X\right)=n \pi w(\exp X) .
\end{gathered}
$$

Theorem 7. Let $\mathbb{A}$ be a subset of $\mathbb{R}$ such that int $(\mathbb{R} \backslash \mathbb{A}) \neq \emptyset$. Then the following nonequalities hold for the Hattori space $(\mathbb{R}, \tau(\mathbb{A}))$ and the functor of permutation degree $S P^{2}$ :

(1) $s(\mathbb{R}, \tau(\mathbb{A})) \neq s\left(S P^{2}(\mathbb{R}, \tau(\mathbb{A}))\right)$;

(2) $h d(\mathbb{R}, \tau(\mathbb{A})) \neq h d\left(S P^{2}(\mathbb{R}, \tau(\mathbb{A}))\right)$;

(3) $h \pi w(\mathbb{R}, \tau(\mathbb{A})) \neq h \pi\left(S P^{2}(\mathbb{R}, \tau(\mathbb{A}))\right)$;

(4) $h \operatorname{sh}(\mathbb{R}, \tau(\mathbb{A})) \neq h \operatorname{sh}\left(S P^{2}(\mathbb{R}, \tau(\mathbb{A}))\right)$;

(5) $h c(\mathbb{R}, \tau(\mathbb{A})) \neq h c\left(S P^{2}(\mathbb{R}, \tau(\mathbb{A}))\right)$;

(6) $h k(\mathbb{R}, \tau(\mathbb{A})) \neq h k\left(S P^{2}(\mathbb{R}, \tau(\mathbb{A}))\right)$;

(7) $h p k(\mathbb{R}, \tau(\mathbb{A})) \neq h p k\left(S P^{2}(\mathbb{R}, \tau(\mathbb{A}))\right)$;

(8) $\operatorname{hpsh}(\mathbb{R}, \tau(\mathbb{A})) \neq h \operatorname{psh}\left(S P^{2}(\mathbb{R}, \tau(\mathbb{A}))\right)$; 
(9) $h w d(\mathbb{R}, \tau(\mathbb{A})) \neq h w d\left(S P^{2}(\mathbb{R}, \tau(\mathbb{A}))\right)$;

(10) $h l(\mathbb{R}, \tau(\mathbb{A})) \neq h l\left(S P^{2}(\mathbb{R}, \tau(\mathbb{A}))\right)$;

(11) he $(\mathbb{R}, \tau(\mathbb{A})) \neq h e\left(S P^{2}(\mathbb{R}, \tau(\mathbb{A}))\right)$.

P r o o f. It is known that the space $S P^{2} X$ contains the squared Hattori space $X^{2}$. However, $X^{2}$ contains a discrete set of cardinality $c$. The other nonequalities can be easily checked. Theorem 7 is proved.

Corollary 7. The functor $S P^{2}$ does not preserve Hattori spaces on the real line.

Corollary 8. Let $\mathbb{A}$ be a subset of $\mathbb{R}$ such that int $(\mathbb{R} \backslash \mathbb{A}) \neq \emptyset$, and let $G$ be an arbitrary subgroup of the group $S_{3}$. Then the following nonequalities hold for the Hattori space $(\mathbb{R}, \tau(\mathbb{A}))$ and the functor of permutation degree $S P_{G}^{3}$ :

(1) $s(\mathbb{R}, \tau(\mathbb{A})) \neq s\left(S P_{G}^{3}(\mathbb{R}, \tau(\mathbb{A}))\right)$;

(2) $h d(\mathbb{R}, \tau(\mathbb{A})) \neq h d\left(S P_{G}^{3}(\mathbb{R}, \tau(\mathbb{A}))\right)$;

(3) $h \pi w(\mathbb{R}, \tau(\mathbb{A})) \neq h \pi w\left(S P_{G}^{3}(\mathbb{R}, \tau(\mathbb{A}))\right)$;

(4) $h s h(\mathbb{R}, \tau(\mathbb{A})) \neq h s h\left(S P_{G}^{3}(\mathbb{R}, \tau(\mathbb{A}))\right)$;

(5) $h c(\mathbb{R}, \tau(\mathbb{A})) \neq h c\left(S P_{G}^{3}(\mathbb{R}, \tau(\mathbb{A}))\right)$;

(6) $h k(\mathbb{R}, \tau(\mathbb{A})) \neq h k\left(S P_{G}^{3}(\mathbb{R}, \tau(\mathbb{A}))\right)$;

(7) $h p k(\mathbb{R}, \tau(\mathbb{A})) \neq h p k\left(S P_{G}^{3}(\mathbb{R}, \tau(\mathbb{A}))\right)$;

(8) hpsh $(\mathbb{R}, \tau(\mathbb{A})) \neq \operatorname{hpsh}\left(S P_{G}^{3}(\mathbb{R}, \tau(\mathbb{A}))\right)$;

(9) $h w d(\mathbb{R}, \tau(\mathbb{A})) \neq h w d\left(S P_{G}^{3}(\mathbb{R}, \tau(\mathbb{A}))\right)$;

(10) $h l(\mathbb{R}, \tau(\mathbb{A})) \neq h l\left(S P_{G}^{3}(\mathbb{R}, \tau(\mathbb{A}))\right)$;

(11) he $(\mathbb{R}, \tau(\mathbb{A})) \neq$ he $\left(S P_{G}^{3}(\mathbb{R}, \tau(\mathbb{A}))\right)$.

Corollary 9. The functor $S P_{G}^{3}$ does not preserve Hattori spaces on the real line.

\section{REFERENCES}

1. Aleksandrov P.S., Fedorchuk V.V., Zaitsev V.I. The main aspects in the development of set-theoretical topology. Russian Math. Surveys, 1978. Vol. 33, No. 3. P. 1-53. DOI: 10.1070/RM1978v033n03ABEH002464

2. Beshimov R.B., Mamadaliev N.K., Mukhamadiev F. G. Some properties of topological spaces related to the local density and the local weak density. Math. Stat., 2015. Vol. 3, No. 4. P. 101-105. DOI: $10.13189 / \mathrm{ms} .2015 .030404$

3. Beshimov R. B. A note on weakly separable spaces. Math. Morav., 2002. Vol. 6. P. 9-19. DOI: 10.5937/MatMor0206009B

4. Beshimov R. B. Some cardinal properties of topological spaces connected with weakly density. Methods Funct. Anal. Topology, 2004. Vol. 10, No. 3. P. 17-22. http://mfat.imath.kiev.ua/article/?id=251

5. Beshimov R. B., Mukhamadiev F. G. Cardinal properties of Hattori spaces and their hyperspaces. Questions Answers Gen. Topology, 2015. Vol. 33, No. 1. P. 43-48.

6. Beshimov R. B., Mukhamadiev F. G., Mamadaliev N. K. The local density and the local weak density of hyperspaces. Int. J. Geom., 2015. Vol. 4, No. 1. P. 42-49. 
7. Engelking R. General Topology. Berlin: Heldermann Verlag, 1989. 529 p.

8. Fedorchuk V. V. Covariant functors in the category of compacts, absolute retracts, and $Q$-manifolds. Russian Math. Surveys, 1981. Vol. 36, No. 3. P. 211-233. DOI: 10.1070/RM1981v036n03ABEH004251

9. Fedorchuk V. V., Filippov V. V. Topology of Hyperspaces and its Applications. Moscow: Mathematica, Cybernetica, 1989. Vol. 4. 48 p. (in Russian)

10. Hattori Y. Order and topological structures of posets of the formal balls on metric spaces. Mem. Fac. Sci. Eng. Shimane Univ. Ser. B: Math. Sci., 2010. Vol. 43. P. 13-26.

11. Michael E. Topologies on spaces of subsets. Trans. Amer. Math. Soc., 1951. Vol. 71, No. 1. P. $152-182$. DOI: 10.1090/S0002-9947-1951-0042109-4

12. Mukhamadiev F. G. Some cardinal and topological properties of the $n$-permutation degree of a topological spaces and locally $\tau$-density of hyperspaces. Bull. Nat. Univ. Uzbekistan: Math. Nat. Sci., 2018. Vol. 1, No. 1. Art. no. 11. P. 30-35. https://uzjournals.edu.uz/mns_nuu/vol1/iss1/11

13. Wagner C. H. Symmetric, Cyclic, and Permutation Products of Manifolds. Warszawa: PWN, 1980.48 p. 